\title{
KNOWLEDGE DICHOTOMY AND SEMANTIC KNOWLEDGE MANAGEMENT
}

Jiehan Zhou

Technical Research Centre of Finland, Kaitoväylä 1, 90571 Oulu, Finland

\begin{abstract}
Today's business is becoming global and knowledge-intensive. This requires business systems capable of knowledge identification, knowledge acquirement, knowledge distribution and knowledge maintenance in terms of universal knowledge understanding. Semantic knowledge management is expected to meet this requirement. This paper presents the fundamentals of semantic knowledge management, including basic concepts, knowledge dichotomy, knowledge solidification modes, and a common semantic knowledge management system.
\end{abstract}

Key words: knowledge management; semantic knowledge management

\section{INTRODUCTION}

Modern businesses are knowledge-intensive. The knowledge-intensive business embodies intensive multi-disciplinary knowledge (e.g. a flying car, a photographing mobile phone), intensive product/service decision-making knowledge (e.g. information on supply, geographical allocation, political and culture factors of production resources), intensive product/service implementation knowledge (e.g. distributed collaborative product design, manufacturing and assembly), and intensive product/service knowledge (e.g. global distribution network, multi-cultural customer psychology and aesthetics). This results in growing research on semantic knowledge management with the use of advanced Internet techniques.

Research on knowledge management systems, e.g. CommonKADS (Schreiber 1994), MIKE (Angele 1998), PROTÉGÉ-II (Gennari 2003) is converting the art and craft of knowledge engineering into a real scientific discipline. The current studies such as semantic Web (Semantic Web 2005), ontology engineering (Gómez-Pérez 2004) and semantic search engine (Corby 2002) are being expected to guide knowledge management towards semantic knowledge management.

This paper purposes to present the fundamentals of semantic knowledge management. It is organized in the following way. Section 2 defines basic concepts. Section 3 studies computer-aided knowledge management, including a general knowledge management model, knowledge dichotomy 
and essentials of computer-aided knowledge management. Section 4 classifies knowledge solidification modes. Section 5 identifies the requirements of semantic knowledge management. Section 6 introduces a common semantic knowledge management system. Section 7 draws a conclusion to the paper.

\section{NOTIONS}

Data, information and knowledge. Data is an uninterrupted signal. A name, phone number, or contact address of a person is one example. Information is data equipped with meaning. For a car provider, a 'Ford' name is not just a brand of some car object; rather, it is interpreted as an indication of a car-making organization. Knowledge is the whole body of data and information leading a community's activities of making things, for example, the 'Ford' culture or phenomenon. Things include material and immaterial. Knowledge is embedded in things, categorized into explicit knowledge and implicit knowledge (or tacit knowledge). Explicit knowledge can be used for making statements of things with a kind of primitive knowledge, namely knowledge standards. Explicit knowledge usually appears in the form of books, manuals, specifications, standards and methods. Implicit knowledge cannot be described in words, is hard to distribute and exists in an individual's brain and a group's values like brief, experience, know-how, credit and culture.

Knowledge in context. In fact, knowledge depends much on context. The context includes concepts, attribute/values, environment setting, inference rules, and the facts in terms of an action. An action out of context might do things right, but might not do right things. For example, young kids might move chess pieces quickly but wrongly; an expert might do product design decision-making better than a layman; a puzzle fan easily thinks out the answer to a riddle in a reasonable context. Knowledge types include concepts, relations, rules and their instances, which are context-dependent.

An ontology is a shared knowledge standard or knowledge model defining primitive concepts, relations, rules and their instances which comprise a topic knowledge. It can be used for capturing, structuring and enlarging explicit and tacit topic knowledge across people, organizations and computer and software systems. We refer to ontology as knowledge ontology.

Knowledge management. Many studies regard knowledge management as a series of interrelated activities of knowledge identification, acquisition, storage, distribution, reuse, maintenance and development. This paper views knowledge management as two main tasks of knowledge standardization and knowledge instantiation.

Knowledge standardization is to capture knowledge types or knowledge ontology for knowledge instantiation. It can be replaced by the term of 
ontology engineering. Knowledge instantiation is to exemplify knowledge types or knowledge ontology.

Knowledge management objectives. Knowledge management views knowledge as a structurable resource. Just as with any other resource management, knowledge management aims to provide the resource in a way of 'at the right time, at the right place, in the right form, to the right knowledge worker, with the needed quality and against the lowest possible costs'.

Semantic knowledge management is a method for obtaining knowledge management objectives with a base of knowledge digitalization and knowledge ontology, which is remarkably distinguished from the way of knowledge management in a human brain.

\section{COMPUTER-BASED KNOWLEDGE MANAGEMENT}

\subsection{General knowledge management}

There are many knowledge management models. Their common intention is to cover the complete life cycle of knowledge within the organization shown in Figure 1. Typically, the following activities with respect to knowledge and its management are distinguished by many authors(Schreiber 1999).

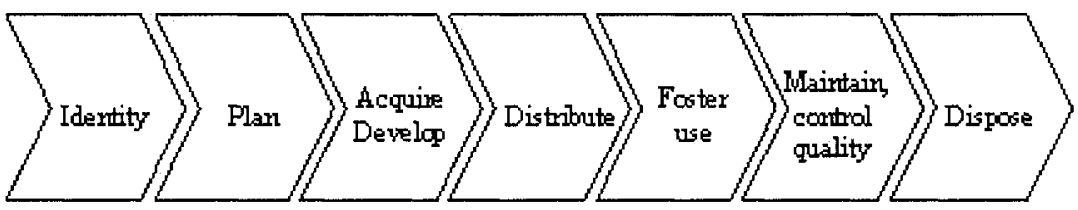

Figure 1. General knowledge management model

- Identify internally and externally existing knowledge

- Plan what knowledge will be needed in the future

- Acquire and /or develop the needed knowledge.

- Distribute the knowledge to where it is needed.

- Foster the application of knowledge in the business processes of the organization.

- Control the quality of knowledge and maintain it.

- Dispose of knowledge when it is no longer needed.

\subsection{Nonaka's Knowledge transformation model}

Nonaka et al. introduced a knowledge transformation model. Four modes are identified as follows (Nonaka 1995): 
- From tacit to tacit knowledge (=socialization): people can teach each other by showing rather than speaking about the subject matter;

- From tacit to explicit knowledge (=externalization): knowledge practices are clarified by putting them down on paper, formulate them in formal procedures, and the like;

- From explicit to explicit knowledge (=combination): creating knowledge through the integration of different pieces of explicit knowledge;

- From explicit to tacit knowledge (=internalization): performing a task frequently leads to a personal state where we can carry out a task successfully without thinking about it.

\subsection{Knowledge dichotomy}

Knowledge management is a kind of human behavior. Human knowledge management is originated for problem solving and passes through the cycle of human survival and evolution. Human problem solving is pervasive and ubiquitous, from the knowledge discovery to the learning of already existing knowledge. Human knowledge activity might be passive or initiative. Initiative knowledge behavior has an obvious goal, for instance, such as question answering and profit earning; passive knowledge behavior does not have an obvious goal, as in the case of knowledge instillation into a baby. In any case, knowledge behavior takes knowledge standards as a basis. This occurs through a knowledge dichotomy: knowledge consists of instantiation knowledge and standard knowledge. In this way, knowledge management consists of knowledge instantiation, knowledge standardization and knowledge evolution.

- Knowledge instantiation. Human beings usually perform knowledge instantiation by taking knowledge standards as foundation and accepting or rejecting data and facts.

- Knowledge standardization. When a human being is unable to describe the facts or data at hand with existing standard knowledge, knowledge standardization is employed. It begins with a comparison with the existing standard knowledge.

- Knowledge evolution. Knowledge evolution is involved in knowledge instantiation, knowledge standardization and a new formation of knowledge instantiation and knowledge standardization with an enlarged knowledge standard.

According to the above standard-based knowledge management, we transcribe Nonaka's knowledge transformation as follows:

- Knowledge socialization and knowledge internalization. Nonaka's knowledge socialization and knowledge internalization are fermentation processes of knowledge standardization, which enables 
human beings to describe knowledge in an intuitive manner, such as expression, gesture and emotion.

- Knowledge externalization and knowledge combination. Nonaka's knowledge externalization and combination is a process of knowledge instantiation, which describes and represents knowledge in the guidance of standard knowledge, for instance, signs, languages and symbols.

Take manufacturing knowledge as an example, manufacturing knowledge management consists of manufacturing knowledge standardization and manufacturing knowledge instantiation. Today's manufacturing behavior is mostly carried out with the guidance of standard manufacturing knowledge. Manufacturing knowledge standardization takes in the research and development knowledge on products and production methodologies. For a 'product-out' (just producing the products) enterprise, it is an important factor for it to customize the existing manufacturing knowledge standard to obtain a high business efficiency.

\subsection{Computer-aided knowledge management}

Knowledge management increasingly plays an important role in human production. The computer is a powerful facility for doing it. A computeraided human knowledge management model is shown as Figure 2.

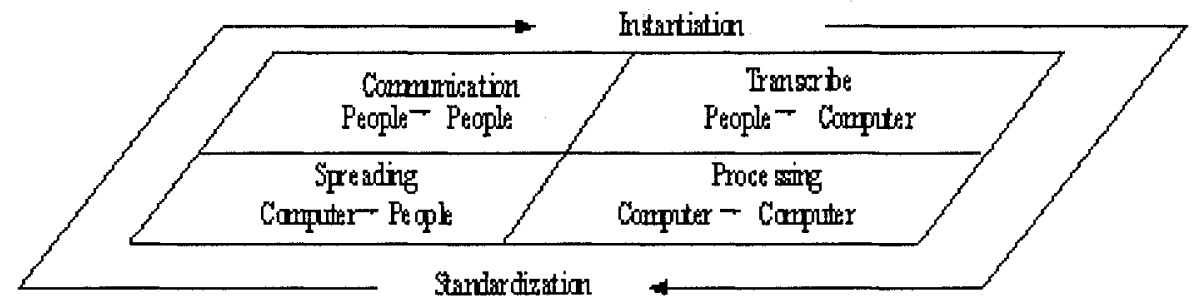

Figure 2. Computer-aided knowledge management

- People to people. It happens when people communicate with each other by natural languages, gestures or expressions. Knowledge standardization takes place gradually in people-to-people knowledge communication. Computer aids for this are not available of this phase

- People to computer. It means people instantiate knowledge with the aid of a computer. An ideal knowledge model facilitates people to exemplify most of the gained knowledge.

- Computer to computer. In which computer stores and processes standard knowledge and instantial knowledge. A new instantial knowledge can be produced by computer inferences.

- Computer to people. Where computer provides people with instantial knowledge and standard knowledge 
Consequently, the main functions of computer-aided knowledge management are computer-aided knowledge instantiation and knowledge standardization. Computer-aided knowledge standardization is a process for discovering a new knowledge model, in contrast to an existing knowledge model. This task mainly takes natural language processing and manual knowledge standardization as the basic means. Computer-aided knowledge instantiation facilitates people to fill knowledge models. A computer-aided knowledge query can be viewed as a reverse process of knowledge instantiation.

\subsection{Matters in computer-aided knowledge management}

Computer-aided knowledge management is an inevitable paradigm coproduced by traditional computer information processing, artificial intelligence and emerging Internet computation. Similar to mechanical devices taking the place of human physical work, computer-aided knowledge management is gradually taking the place of human mental work, for instance, knowledge memory and knowledge discovery. It contains the following four important matters:

- Partly freeing human from mental work. Computer-aided knowledge management keeps people concentrating on creative mental work. In other words, it facilitates people to develop knowledge standards.

- Enlarging the scope of knowledge reusing and sharing. Computeraided knowledge management not only accelerates binary-coded knowledge spreading, but also distributes semanticized knowledge.

- Reducing knowledge management costs. The cost of standard-based knowledge management will be far lower than non-standard-based type. Computer-aided knowledge management is based on knowledge standards or knowledge models.

- Unifying knowledge processing. People and software process knowledge in conformance to a unified knowledge model, which enhances knowledge evolution.

\section{KNOWLEDGE SOLIDIFICATION MODES}

Knowledge management accompanies knowledge solidification, namely structuring knowledge. Knowledge solidification has extended over several thousand years since humans came into being. For instance, the simplest knowledge solidification is to remember things with physical brains. Along with the increasing development of computing techniques, it changes profoundly in knowledge solidification. This paper discusses three kinds of important knowledge solidification modes: human brain-based, paper-based and computer-based modes. The computer-based mode can be classified into four types: data structure-based, entity-relation, object-oriented and semantic-based mode. The computer-based mode, paper-based mode and 
human brain-based mode have their advantages and disadvantages respectively. For example, the accuracy in the computer-based knowledge query is superior to the human brain-based one. However the computerbased is unable to take the place of the human brain in knowledge evolution forever. The semantic-based type in the computer-based mode is more efficient in problem solving than the entity-relation type. They are all developed by these essential ideas of knowledge sharing and semantic-based knowledge modeling. The scope of knowledge sharing mainly distinguishes these methods. Table 1 presents a summary of the three knowledge solidification modes.

Table 1. Summary of knowledge solidification modes

\begin{tabular}{|l|l|l|l|l|l|}
\hline Mode & $\begin{array}{l}\text { Knowledge } \\
\text { source }\end{array}$ & $\begin{array}{l}\text { Knowledge } \\
\text { type }\end{array}$ & Readability & $\begin{array}{l}\text { Under- } \\
\text { standability }\end{array}$ \\
\hline Brain-based & $\begin{array}{l}\text { Knowledge } \\
\text { owner }\end{array}$ & Richest & Only human & Only human \\
\hline Paper-based & $\begin{array}{l}\text { Paper } \\
\text { documents }\end{array}$ & Richer & Only human & Only human \\
\hline $\begin{array}{l}\text { Computer } \\
\text {-based }\end{array}$ & $\begin{array}{l}\text { Data } \\
\text { structure }\end{array}$ & $\begin{array}{l}\text { Data } \\
\text { structure }\end{array}$ & $\begin{array}{l}\text { Application } \\
\text {-specific }\end{array}$ & $\begin{array}{l}\text { Programmer } \\
\text {-specific }\end{array}$ & $\begin{array}{l}\text { Programmer- } \\
\text { specific }\end{array}$ \\
\cline { 2 - 6 } & E-R & Database & $\begin{array}{l}\text { Enterprise } \\
\text {-specific }\end{array}$ & $\begin{array}{l}\text { Intra } \\
\text {-enterprise }\end{array}$ & Intra-enterprise \\
\cline { 2 - 6 } & O-O & Class base & $\begin{array}{l}\text { Programmer } \\
\text {-specific }\end{array}$ & $\begin{array}{l}\text { Programmer } \\
\text {-specific }\end{array}$ & $\begin{array}{l}\text { Programmer- } \\
\text { specific }\end{array}$ \\
\cline { 2 - 6 } & Semantics & $\begin{array}{l}\text { Knowledge } \\
\text { base }\end{array}$ & $\begin{array}{l}\text { Domain } \\
\text {-specific }\end{array}$ & $\begin{array}{l}\text { Domain } \\
\text { community }\end{array}$ & $\begin{array}{l}\text { Domain } \\
\text { community }\end{array}$ \\
\hline
\end{tabular}

\subsection{Physical brain-based mode}

Brain-based knowledge solidification is an effort of obtaining right knowledge in a process of gathering, transcribing and analyzing manufacturing expert's knowledge. According to the expert division in (Schreiber 1999), the three types of academic, practitioner and operator are distinguished. There are knowledge acquisition techniques of interviews, brainstorming and discussions usually used in the brain-based mode. The acquired knowledge is confined to the on-site experts. But there is more to say about the nature of experts that is rooted in the general principles of human information processing. Psychology has demonstrated the limitations, cognitive biases, and prejudices that pervade all brain-based knowledge acquisition. Considering this evidence, it is possible that experts may not have access to the same information when in a knowledge acquisition interview as they do when actually performing the task.

\subsection{Paper-based mode}

Paper-based knowledge solidification explores the right knowledge by reading, marking up and annotating technical documents. The knowledge source may be in one of the possible forms of messages. text files. paper 
books, manuals, notes, etc. Unlike individual experts, documents hardly contain very practical know-how acquired through experience. Indeed, they are a consensual view on the domain. Before hard copy-based knowledge acquisition can be taken, the knowledge worker must be sufficiently acquainted with the domain, and the required documents must be accessible.

\subsection{Computer-based modes}

Computer-based mode refers to that kind of knowledge acquisition in which both an expert's knowledge and document knowledge are formalized in a digital form by means of data modeling. The computer-based mode is categorized as data structure-based, entity-relation-based, object-oriented and semantic-based modes. The borderlines between them are not sharp, because they are relative in terms of context.

- Data structure-based mode. In this mode, knowledge is held in a structured manner that adheres to a well-defined model. This model may be proprietary, however, we are increasingly seeing the appearance of common models that adhere to ISO standards e.g. STEPs(NIST 2005). For example, a geometric wireframe entity is presented by points, lines, and arcs.

- The Entity-Relation-based mode uses enterprise databases as the knowledge source. Entity-Relation (ER) is used as the main method for designing enterprise databases. The ER method views the real world as entities and relations. The basic ER components are entities, relationships, attributes etc.

- Object-Oriented mode. In this, knowledge is written and acquired in terms of real-world objects, classes, subclass/super class, attributes, not internal data structures. This makes knowledge somewhat easier to understand by maintainers and people who have to read the knowledge code.

- The semantic-based mode helps knowledge workers set up complex analyses and structuring of knowledge acquisition. Knowledge users capture knowledge in the context of definitions of concepts, relations and rules, and instances.

\section{REQUIREMENTS ON SEMANTIC KNOWLEDGE MANAGEMENT}

Many new requirements are proposed for semantic knowledge management as follows:

- Manufacturing knowledge sharing and reusing. Abilities to share and reuse manufacturing knowledge are the primary requirements in developing manufacturing knowledge management system.

- Manufacturing knowledge types. More rich knowledge types are required for manufacturing enterprises to customize production 
knowledge due to the transition from 'product-out' and 'market-in' to 'knowledge innovation' in manufacturing business. Current manufacturing knowledge types are flat and specified to applications to a certain extent.

- Knowledge quality. It is important for enterprises to get qualified knowledge. The accuracy plays a key role in weighting knowledge quality. The knowledge accuracy refers to the rightness for an obtained knowledge to the true knowledge (EPISTLE 2005). The two preconditions of improving knowledge accuracy are: knowledge standardization and knowledge instantiation.

- Knowledge cost. Knowledge cost is the total expenditure of knowledge standardization, knowledge instantiation and knowledge evolution (EPISTLE 2005). The cost in human brain-based mode rises quickly during the post-stage of knowledge management, but the cost in semantics-based mode decreases and tends to be steady in the post-stage of knowledge management.

- Knowledge timeliness. The increasingly competitive business requests manufacturing enterprises not only to obtain the accurate knowledge, but also to get it without delay (EPISTLE 2005).

- Knowledge unification. Knowledge unification is the availability of a clear and shared definition for the knowledge. Knowledge unification allows knowledge community to spread and create knowledge quickly.

\section{A COMMON SEMANTIC KNOWLEDGE MANAGEMENT SYSTEM}

Modern industry communities are respectively driven by knowledgeextensive business. The right knowledge is required for knowledge workers to solve problems rightly, not right now. How to accomplish business knowledge standardization and knowledge instantiation are two key points in realizing semantic knowledge management. Knowledge query could be done by manual means and Internet means. Textbook reading and expert consultation are two examples of the manual one. The manual knowledge query costs much. The knowledge quality is decided by the expert competency and textbook writers. Nowadays, there is an inadequacy in electronic manufacturing documents (e.g. plain and flat Web page) and knowledge search techniques (e.g. plain and flat query interface) for Internet knowledge query. These are incapable for meeting the needs of knowledge timeliness and knowledge accuracy. Knowledge instantiation is another point in modern knowledge management. Human brain-based and paperbased knowledge instantiation are two main ways for knowledge storage, in which it is difficult and costly to convey and renew knowledge. 
Conventional knowledge evolution is done by creating new manufacturing concepts, rules, and relations manually.

A common semantic knowledge management approach is a semanticsbased knowledge solidification system running over the Internet for managing and developing manufacturing knowledge, which consists of the three functions of knowledge standardization, knowledge instantiation and knowledge query as shown in Figure 3.

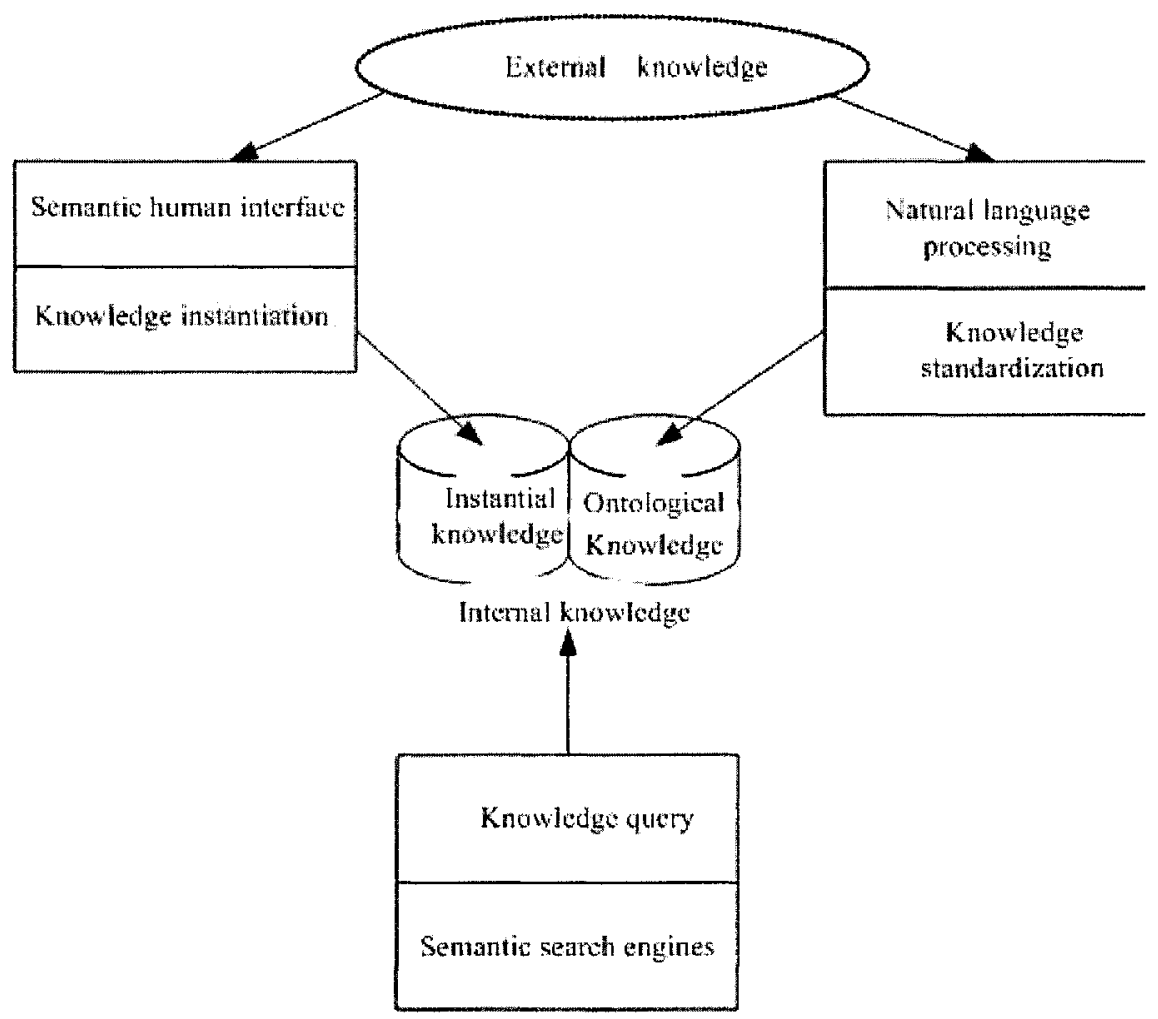

Figure 3. A common semantic knowledge management model

- Knowledge standardization is a computer-aided knowledge modeling process. Take manufacturing knowledge models as an example. There are many standardized manufacturing knowledge models (e.g. STEP (NIST 2005), BPEL4WS (BPEL4WS 2005), EPISTLE (EPISTLE 2005)), which are references for extending manufacturing knowledge model (e.g. new concepts, new rules, and new relations).

- Knowledge instantiation is a computer-aided process of making knowledge instances. Semantic knowledge management provides knowledge workers a semantic human-computer interface for customizing knowledge models. entering a new knowledge instance 
entry. If the outward knowledge is capable of being inserted into a knowledge base, it is completely decided by the richness of the existing manufacturing knowledge model.

- Knowledge query is a computer-aided process of acquiring standard knowledge and instantial knowledge. Compared with a plain keywords-based search interface, a semantic knowledge query is advantageous in accurate and proper knowledge query with customized manufacturing knowledge types available.

\section{CONCLUSION}

Global commercial demands are promoting a growth in the reseach of semantic knowledge management. This paper presented the fundamentals of semantic knowledge management, including basic concepts, computer-aided knowledge management, knowledge dichotomy, knowledge solidification modes, requirements of semantic knowledge management and a common semantic knowledge management system. These fundamentals were successfully applied to implement a semantic manufacturing knowledge management system (Zhou 2004a; Zhou 2004) .

\section{ACKNOWLEDGEMENTS}

Thanks to Prof. Rose Dieng for her advices and thanks to ERCIM (the European Research Consortium for Informatics and Mathematics) organization for the financial support.

\section{REFERENCES}

Angele, J., et al. (1998). "Developing Knowledge-Based Systems with MIKE." Journal of Automated Software Engineering 5(4): 389-418.

BPEL4WS (2005). Business Process Execution Language for Web Services. http://xml.coverpages.org/ni2003-04-16-a.html

Corby, O., Faron, C. (2002). Corese : A corporate semantic Web engine. I1 th International World Wide Web Conference Workshop on Real World RDF and Semantic Web Applications, Hawai, USA.

EPISTLE (2005). Process Industries Data Handover Guide. Part 1 and Part 2. http://www.epistle.ws/

Gennari, J. H. e. a. (2003). "The Evolution of Protege: An Environment for KnowledgeBased Systems Development." International Journal of Human-Computer Studies.

Gómez-Pérez, A., et al. (2004). Ontological engineering. London, Springer Verlag.

NIST (2005). STEP Online Infermation Server. http://www.nist.gov/sc4/

Nonaka, I., H.Takeuchi (1995). The Knowledge Creating Company, Oxford University Press.

Schreiber, G. e. a. (1999). Knowledge Engineering and Management: The CommonKADS Methodology, MIT Press.

Schreiber, T., et al. (1994). "CommonKADS: A Compre-hensive Methodology for KBS Development." IEEE Expert: 28-37.

Semantic Web, W. C. (2005). Semantic Web News and Events. http://www.w3.org/2001/sw/ 
Zhou J., Dieng R. (2004a). Manufacturing Ontology Analysis and Design: towards Excellent Manufacturing. INDIN'04 2nd IEEE International Conference on Industrial Informatics, Berlin, Germany.

Zhou J., Dieng R. (2004). A Semantic knowledge management system for knowledgeintensive manufacturing. 2004 IADIS International Conference of e-Commerce, Lisbon, Portugal. 